\title{
CAUSAL INFERENCES OF EXTERNAL-CONTEXTUAL DOMAINS ON COMPLEX CONSTRUCTION, SAFETY, HEALTH AND ENVIRONMENT REGULATION
}

Nnedinma Umeokafor ${ }^{\mathrm{a}}$, Abimbola Windapo ${ }^{\mathrm{b}}$, Konstantinos Evangelinos ${ }^{\mathrm{c}}$

${ }^{a}$ School of Design, Faculty of Liberal Arts and Sciences, University of Greenwich, United

Kingdom

${ }^{\mathrm{b}}$ Department of Construction Management, University of Cape Town, South Africa

${ }^{c}$ Department of Environment, University of the Aegean, Lesvos, Greece

\section{ABSTRACT}

A robust and pragmatic regulatory framework that is based on a good understanding of the external-context domains of countries is fundamental for Safety, Health and Environment (SHE). However, in many developing and emerging economies the regulatory framework for SHE is complex and the external-context domains are poorly understood and not factored in SHE. Using Nigeria as a case, the study examines the causal inferences of the social, cultural, political, religious and institutional contexts on the complex Construction Safety, Health and Environment (CSHE) regulatory framework using a qualitative research approach. The findings show that the external-context domain factors are indirect determinants of CSHE regulation. There is evidence that the main external-context factors include the dysfunctional and fragmented health and safety (H\&S) regulatory environments, which is exacerbated by the poor governmental and political attention on H\&S. While political influence results in the low threat of regulation, 'Nigerian factors' such as 'the no follow-up culture' result in inadequate governmental and political involvement, among many, poor regulation and inadequate H\&S laws. Although the need for a consolidated CSHE regulatory framework is emphasised hence recommended, it should be resilient to social and political pressure.

Keywords: Construction safety, Cultural context, Social context, Political context, Well-being.

\section{INTRODUCTION}

Globally, construction industries are hazardous and record high levels of fatal injury (Health and Safety Executive (HSE) 2018; International Labour Organisation (ILO) 2017; Manu et al. 2018; Muiruri and Mulinge 2014; Okoye 2018), up to four times as high as the average across other industries (HSE 2018). Despite the dearth of statistics, Manu et al. (2018) empirically demonstrate that levels of fatal injuries in the construction industries of Emerging and Developing Economies (EDE) are worse than that of developed economies.

However, efforts towards improving the Construction Safety, Health and Environment (CSHE) in EDEs have 'yielded fruits' but below expectation. For example, there is evidence of improved health and safety (H\&S) including implementation of $\mathrm{H} \& \mathrm{~S}$ practices in EDEs such as Vietnam where two consecutive 5year H\&S programmes have been implemented since 2006 (Manu et al. 2018; Ministry Of Labour, Invalids and Social Affairs (MOLISA) 2012). These improvements are not limited to a $9 \%$ reduction in the rate of fatal accidents in the construction industry from 2006 to 2008 (MOLISA 2012). In developed (high 
income) economies such as Britain, improvement in CSHE has been reported (HSE 2018).

One of such H\&S improvement is in the regulation of CSHE, the fundamental without which other effective H\&S measures (including the innovative measures such as integrating H\&S in the decision making of organisations) would be ineffective or even non-existent (Finneran and Gibb 2013). H\&S regulation has emerged a key barrier to H\&S improvement in many EDEs unlike most developed countries. For instance, according to Muiruri and Mulinge (2014), in Kenya, while the Occupational Health and Safety Act (OSHA) covers the construction industry, the enforcement is low or not even carried out. Muiruri and Mulinge (2014) conclude that the enforcement mechanism is weak and inadequate resulting in owners/managers taking advantage of this. In Jordan, despite the presence of H\&S laws, Alkilani et al. (2013) found government regulations, legislation and laws including adequate laws for the construction industry, as the main barriers to $H \& S$ practices in the construction industry. In Ghana, Kheni (2008) found poor enforcement and compliance with OHS standards, lack of government commitment to H\&S in their projects. While in South Africa, Windao (2013) identified lack of knowledge and cost mitigation as reasons for non-compliance to H\&S regulations. In Nigeria, the regulation of CSHE is poorly understood and unexamined, and the lack of governmental attention to H\&S remains significant (Umeokafor 2015). In particular, this industry has been reported as unregulated because Section 87 of the Factories Act CAP F1 L.F.N 2004 (one of the main OHS laws in Nigeria) omits construction sites and activities in its definition of premises (see Diugwu et al. 2012; Idoro 2011; Umeokafor et al. 2014).

However, drawing on the definition of self-regulation where the development or adoption of standards and administration is self-imposed with little or no external involvement (Gunningham 2011; Gunningham and Rees 1997), the Nigeria construction industry is self-regulated in various ways on CSHE. Specifically, the adoption and enforcement of H\&S by contractors at their discretion (Idoro 2011) is voluntary self-regulation (Gunningham 2011). The same applies to the adoption of the National Building Code of 2006 by contractors, which as at the time of the current study has no legal backing (Omeife and Windapo 2013). In Lagos State, Nigeria, the Lagos State Safety Commission oversees H\&S in the state and enforces the Lagos State Commission Law 2011 (ILO 2017). The oil and gas sector has H\&S standards and requirements to which construction contractors who work for them must adhere; this is conceptualised as the industry as self-regulation (Umeokafor and Isaac 2015). This is in addition to the Minerals Oils (Safety) Regulations and the Petroleum (Drilling and Production) Regulations 1997 that empowers the Department of Petroleum Resources to oversee HSE in the oil industry of which construction contractors that work in this industry are covered. The regulatory activities of regulatory authorities (such as Federal Ministry of Environment and National Environmental Standards and Regulations Enforcement Agency (NESREA) also cut across the construction industry, just as the Employee Compensation Act 2010 implemented by the Nigeria Social Insurance Trust Fund Management Board. These do not only show the fragmented regulatory system but also demonstrates that there are multiple regulatory actors, interests, structure, methodologies and inadequate H\&S laws. This emphasises the complexity in the regulatory system which according to Abubakar (2016) is the Distributed Occupational Safety and Health (DOSH) Regulatory framework, a characteristic of the regulatory framework of EDEs. 
The complexity is exacerbated by the lack of understanding of social, political, institutional, cultural, religious, and technological contexts in the EDEs (Kheni et al. 2010; Nuwayhid 2004; Umeokafor, 2015). According to these authors, without understanding and considering these in H\&S strategies, the solutions to H\&S issues in developing countries may be unlikely. A lot of strategies, policies and legislation adopted from developed countries are impractical in EDEs (Umeokafor et al. 2018). Nuwayhid (2004) strongly argues that the focus of occupational health research and strategies should be from the "external-contextual domain" to the "internal domain"-from addressing the social and political environment before particularities of the workplace. In other words, focusing on and understanding the externalcontextual domain in $\mathrm{H} \& \mathrm{~S}$ is fundamental and should be first. For example, the Construction Design and Management (CDM) Regulation 2015 stipulates duties for many stakeholders such as clients, designers and contractors (HSE 2015). One of the duties is that clients should ensure that the project is managed to ensure the H\&S of all in the project and the public. One of the ways that the client can do this is by providing pre-construction information to the designers and contractors and providing funds for $H \& S$. These duties do not only enable the other parties such as designers to design for safety - eliminating, controlling or reducing foreseeable risk in the project life cycle including during construction - their duties under regulations (HSE 2015). When these regulations are adopted in EDEs such as Nigeria, clients just like designers and contractors are not obliged to comply. However, if designers or contractors decide to comply but the clients do not comply, for example by providing the preconstruction information to relevant persons, the designers' responsibilities such as designing for safety will be farfetched.

This area - Contextual environments and H\&S - just like many other areas of H\&S in EDEs, has received little attention (Kheni 2008; Kheni et al. 2010; (cf. Manu et al. 2018; Umeokafor 2018a; Umeokafor et al. 2018). In an extensive review of 6241 Built environment/construction management publications over 36 years in Nigeria, Umeokafor (2018) shows that only two publications addressed contextual matters in CSHE. Further, the complexity in the regulation of CSHE in countries such as Nigeria also remains unexamined; the effects of the contexts of developing countries on H\&S regulation is poorly understood as suggested by Umeokafor (2018).

Following this, this paper-part of an extensive study-explores and explains the contextual effects on CSHE regulation including the factors towards understanding the complex regulatory environments of CSHE in EDEs from the Nigeria perspective- The next section is the context of the study, followed by the methodology. The discussion follows the results after which is the concluding remarks, the last section.

\section{CONTEXT}

Nigeria shares borders with the Republics of Benin, Cameroon, Chad, and Niger with a landmass of about 023,760 sq. KM, the most populous country in Africa (ILO 2017)_about 190 million people. It has 36 states and six Geopolitical Zones (GZ), South South, South West, South East, North Central, North West, and North East. In being a democratic state, the arms of government are executive, legislature and judiciary. While it has over 500 ethnic groups; according to Worldatlas (2018), Igbos are $18 \%$, Hausas are $25.1 \%$, Fulani, 3.9\%, Yoruba, 21\%, Ijaw 10\%, Kanuri 4\%, Ibibio 
$3.5 \%$, Tiv $2.5 \%$, other groups make up the remaining $12 \%$. Umeokafor et al. (2018) conclude that the distribution of religion in Nigeria is uncertain, but drawing on Pew Forum (2010) Christians are 46\%, Muslims are 52\%, and other religions are $2 \%$.

The above does not only show that Nigeria is a religious country, but also that the population is diverse. However, according to Hofstede (2001), just like many EDE including West African Countries, it belongs to the collectivism cultural dimension, the contrast of individualism. The features of the collectivism cultural dimension include closer ties, concern about the needs of others (Darwish and Huber 2003; Hofstede 2001), loyalty to the group is a priority over that of individuals (Darwish and Huber 2003). This explains another characteristic, extended family system which is inherent in this cultural dimension (Kheni 2008). This may also explain Kheni's (2008) and Umeokafor's (2018) findings where family values make contractors (as in Kheni 2008) in Ghana and Communities (as in Umeokafor 2018b) in Nigeria to contribute to H\&S. Based on this, in the current study family values is beyond the nuclear family, communities have closer ties, viewing themselves as a big family. This does not preclude the nuclear and extended family system.

Issues of bribery and corruption are well reported in EDEs. For example, Kheni (2008) found that H\&S enforcement officers are compromised in Ghana when offered money to overlook H\&S breaches, Umeokafor (2018b) reports the same in Nigeria. This may explain the discrimination in H\&S enforcement in Ghana that Kheni (2008) also found and power relationship - where the ability of people to control or influence others - in Nigeria that Okojie (2010) reports. People in the high echelon of the society are treated with bias in their favour (Kheni 2008). Typically, according to Okojie (2010), enforcement activities are handicapped by people in the high echelon of society. The unethical practices among organisational leaders in EDEs such as Nigeria are found to be encouraged by environmental pressure, the culture of bribery, among many, stringent policies on bonuses and allowances (Odole 2018).

Despite the above, Umeokafor et al. (2018) find a lot of H\&S improvement opportunities in the external-context domains of EDEs such as Nigeria. Specfically, Umeokafor et al. (2018) demonstrate how, the ability of youth to effect change through the social media, the moral stance of religion, the cultural values, among others, the voluntary individual efforts (of which some are covered in the introduction) can be exploited to improve $\mathrm{H} \& \mathrm{~S}$.

\section{METHODOLOGY}

Creswell (2009) and Saunders et al. (2009) posit that quantitative methods and strategies are concerned with providing a generalised view of a phenomenon, measuring and, among many, testing of hypothesis. However, the current study aims to explore and explain complex socio-legal, socio-cultural, socio-economic and sociotechnical phenomena. In other words, it seeks to establish, advance or gain in-depth understanding or meaning of such phenomena (Saunders et al. 2009; Kheni 2008; Umeokafor 2018a). Consequently, qualitative methods-semi-structured interviews and thematic analysis - instead of quantitative methods and strategies were adopted for the study. Qualitative methods and strategies are appropriate for exploring and providing an understanding of the meanings of social phenomena (Isaacs, 2014). They excel in answering 'how'; 'what' and 'why' research questions (Erikkson \& 
Kovalainen, 2008), which are consistent with the research questions of the extensive study.

While using qualitative research to address causality including explaining has been debated for some time (Maxwell 2004), it is adopted in studies and accepted that qualitative research could be used for making causal inferences or explanations without losing any of its characteristics (Maxwell 2004; Lukka 2014; Plumper et al. 2010). Two examples of such studies are Gutiérrez et al. (1995) and Alggaia and Millington (2008). Gutiérrez et al. (1995) used grounded theory to analyse interviews of administrators and staff of six agencies. The findings of the study show causal inferences such as '...differing philosophies or politics of more traditional service providers negatively affects the willingness or ability of empowerment-based agencies to refer clients to other services' (Gutiérrez et al. 1995: 252). Lukka (2014) argues that using qualitative research for causal inference or explanation maximises the potentials and strengths of qualitative research. In doing this, Lukka (2014) goes on to argue that '...we do not need to seek law-like generalisations and apply the covering law model'. Rather, in agreement with Kakkuri-Knuuttila et al. (2008), Lukka (2014) argues that '...causal explanations capture (difference-making) dependency relations between things in the world in any locality'. Scholars such as Maxwell (2004) Lukka (2014) and Plumper et al. (2010) offer a treatise on the subject of using qualitative research to make causal inferences or explanations.

The current study is concerned with underexplored and salient areas in developing countries, which according to Umeokafor and Windapo (2018) and Kheni (2008) present unique opportunities for qualitative methods and strategies. Further, the contextual features of the study area, for example, the complex nature of the challenges, the characteristics of the social and cultural contexts of DCs, to name but a few, contribute to making a case for the adopted methods (Umeokafor and Windapo 2018 and Kheni 2008). The adopted approach enable the 'close collaboration with industry partners, ... solving practical problems and generating new knowledge in the form of systems, models, or frameworks' (AlSehaimi et al., 2013: 411). According to AlSehaimi et al. (2013) and Umeokafor and Windapo (2018), the adoption of research methods and strategies that are not 'fit for purpose' has exacerbated the unsolved construction management research problems in developing countries. Figure 1 is a graphic presentation of the research

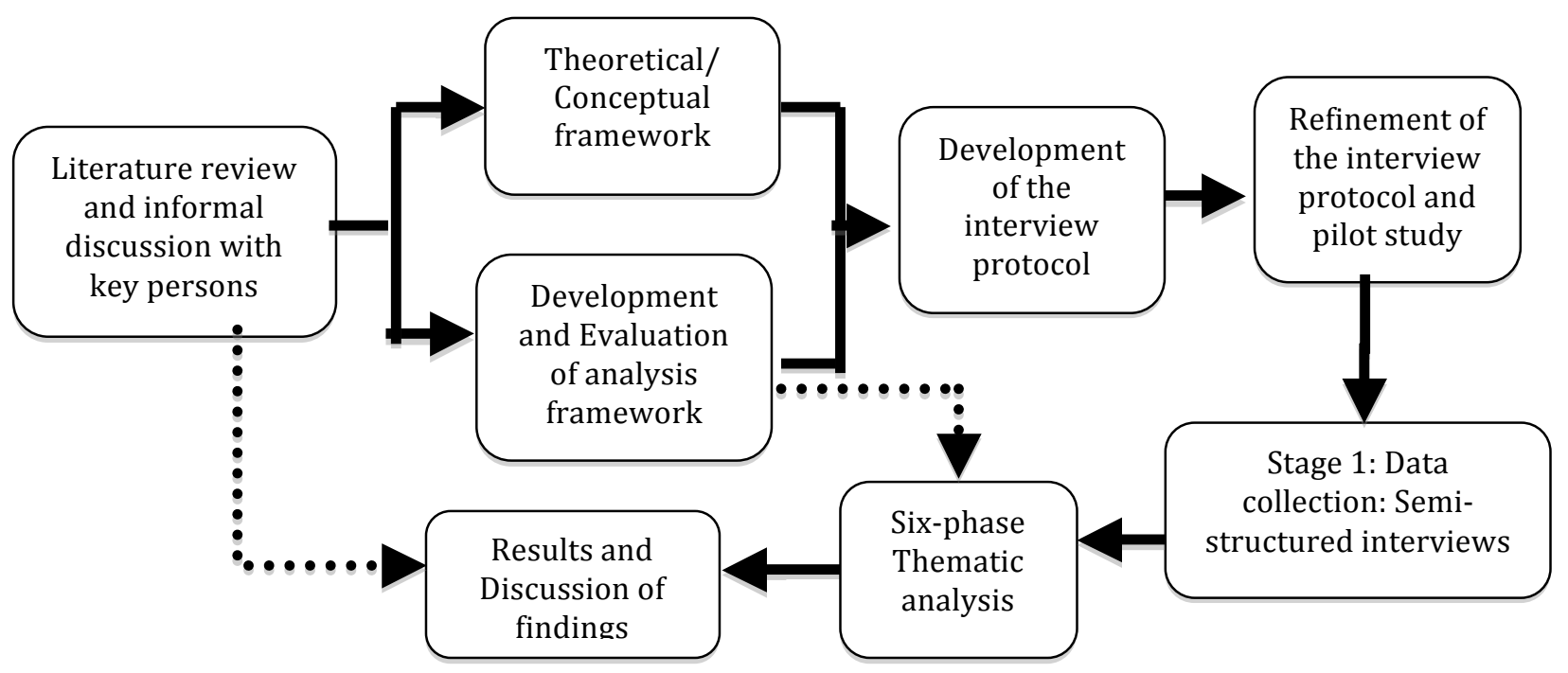


Legend: Analysis link

Process link:

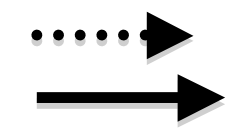

Figure 1: Relevant Research Process; Author's fieldwork

\section{Data collection instrument and the trustworthiness of the research}

The interview protocol was designed based on informal discussions with key informants and the review of relevant literature not limited to cultures (including social norms and values) in Nigeria, institutional environment, religious context, the construction industry (including the methods of construction and challenges) and the $\mathrm{H} \& \mathrm{~S}$ regulatory environment. The semi-structured interview protocol covered various areas including the demographics of the respondents and their organisations, questions on the political, cultural, institutional and social contexts of Nigeria and how they affect the regulation of CSHE. In ensuring that the interview protocol was useable and achieved the aim, was refined using Castillo-Montoya's (2016) fourphase interview protocol refinement framework. This also improved the quality of the research data (Castillo-Montoya 2016). The four phases of the interview refinement protocol are: Phase 1: Ensuring interview questions align with research questions; Phase 2: Constructing an inquiry-based conversation; Phase 3: Receiving feedback on interview protocols; and Phase 4: Piloting the interview protocol.

In line with this, for the first phase, the interview questions were written down and checked against the research questions. This showed the research questions that are addressed by too much interview questions and those addressed with less (CastilloMontoya 2016). They were then adjusted, revised and new questions added where possible. Castillo-Montoya (2016: 831) shows how this approach is not 'mechanically creating interview questions from the research questions without attention to the contexts shaping participants' lives...'; Hence should not be misconstrued as doing so. For the second phase, constructing an inquiry-based conversation, a balance was found established between an inquiry and a conversation, termed inquiry-based conversations by Castillo-Montoya (2016). The questions were short, clear and understandable but conversational (Castillo-Montoya 2016). In this phase, steps such as the possible follow-up questions and prompts were considered and drafted as were the social rules in daily conversations including avoiding judgmental questions such as 'why' question and asking only one question at a time. To improve the reliability of the interview protocol, feedback was sought on the interview protocol-Phase 3. Here, the lead author read the interview protocol aloud and sent it to peers to read aloud. The aim here is to see how difficult answering the interview questions are and how the questions flow. The last phase involved sending the interview protocol to eight persons (including two H\&S experts and academics at the University of Greenwich, two H\&S managers in Nigeria, one experienced Civil Engineer in a government department in Nigeria) for pretesting. After this, the interview protocol was revised and piloted on five Nigerian contractors and four key informants. Based on the outcome, the interview guide was revised.

Other steps to improve the trustworthiness of the research are not limited to triangulation, peer debriefing, thick, rich description, and addressing investigator 
effects. In particular, one of the ways of achieving external validity is a thick, rich description (Lincoln and Guba 1985) where a detailed account of how the research was conducted is provided to enable the readers to make an informed judgment on the transferability of the conclusions and finding of the study to a different context. To address investigator effects, various steps were taken. Specifically, reflectivity (Creswell and Miller 2000) - the lead author consciously acknowledged any beliefs, views and experiences which may affect the data collection and analysis. Concerning triangulation - the use of multiple sources of data collection techniques in a study (Saunders et al. 2009) — this involved multiple triangulation (Adami 2005; Humble, 2009). Here, analytical triangulation and method triangulation were adopted. The methods triangulation was computer-aided analysis with NVivo for Mac and constant comparison of the analytical groups. The analytical groups are the 'Constantly Selfregulating Contractors (CSRCs)', 'Non-Constantly Self-regulating Contractors (NCSRCs)', 'Non-Self-regulating Contractors (Non-SRCs)' and key informants. The person triangulation was the use of the four categories of contractors and the key informants. This was informed by literature review and informal discussions with people in the construction industry. The findings enabled 'within group' analysis and 'between groups' analysis, enabling analytical triangulation in five ways, namely complementary, convergent, dissonant, provided 'unique information' and 'illumination' (Sands and Roer-Strier 2006).

\section{Sampling and data collection}

One key difference between qualitative methods and quantitative methods is the logic and techniques behind the sampling approaches (Patton 1990). While in quantitative methods, the sampling depends on larger sizes selected randomly, in qualitative methods, the sampling focuses on smaller sampling as little as one, selected purposefully (Patton 1990). In probability sampling, the logic and power are selecting a sample that is truly random and statistically representative of the population towards generalisations, but the purpose and power of purposeful sampling is in selecting 'information-rich cases' for the study. Information-rich cases are those that can provide significant information on the object of study (Patton 1990). According to Palys (2008), the sampling strategy depends on the context of research and the research questions. Consequently, information-rich cases can be selected through different strategies (Patton 1998). In other words, an adequate sampling strategy is context-defined (Palys 2008).

Drawing on this, to address the objectives, the study adopted a combination or purposeful mixed sampling of stratified purposeful sampling and snowball sampling techniques. The stratified purposeful sampling helped ensure variation or characteristics of the population (Patton 1998). Covering the six GZ of Nigeria, the four categories of contractors, Large (LCs), Medium (MCs), Small (SCs) and Micro (MiCs), were selected to cover the strata. This is a presentation of the full breadth of the population and not the statistical representation (Adami 2005). The criteria for this is that LCs employ over 199 workers, MCs employ from 30 to 199 workers, SCs employ from 10 to 29 workers and MiCs employees below ten workers. Regarding the snowball sampling, the participants - key informants - were those that have a direct or indirect association or relationship with the construction industry, for example, employees of regulatory authorities whose activities cut across the construction industry, a lawyer and an employee of a trade association. 
The University of Greenwich Research Ethics Committees approved the research including the data collection instruments and process. The participants were sent introductory letters that informed them of how the data will be used, assured of anonymity, how sensitive information will be used, among others, the options of withdrawal at various stages of the data collection process. Of 53 interviews conducted, the 46 used comprised of 31 contractors, 15 key informants from 13 institutions. The interviews which lasted 67-117 minutes were conducted face-to-face and telephonically. Field notes were taken during the interviews and analysis. Before each interview, the respondents were reminded of ethical considerations such as the options of opting out of the interview, anonymity and how the data will be used. The interviews were recorded with the permission of the respondents and transcribed verbatim. During the face-to-face interviews, the lead author maintained eye contact, and also observed the respondents, which according to McCracken (1988) is inevitable. The interviewees were made to relax before the interviews. The questions were probed through many ways such as suggesting but not leading the interviewees and asking the respondents to expand on responses where relevant.

\section{Data analysis}

Using NVivo for Mac, the data was analysed thematically by exploring the latent and manifest meaning till saturation. This was achieved with the saturation grid by Brod et al. (2009) hence not all conducted interviews were used. The thematic analysis was a combination of deductive and inductive approaches - the hybrid approach consistent with Fereday and Muir-Cochrane (2006); inductive approach (Braun and Clarke 2006) and deductive approaches. Each of the three was used for various objectives or research questions of the extensive study. The Inductive approach involves coding the data to fit a preexisting code frame. This can also be an analytical preconception of the analyst, which can be informed by literature; however, in deductive approach, the coding is theory driven. Braun and Clarke (2006) proposed the six-phase step-by-step guide for thematic analysis; just like other analysis processes, it is not linear, but unlike some, it is flexible. The six phases of analysis used are: familiarisation with the data; generating initial codes; developing or searching for themes; reviewing themes; defining and naming themes; and writing up.

Familiarisation with the data: despite the initial thoughts and field notes during the interviews, the lead author read the transcripts many times to be immersed in the data. The note taking continued at this stage.

At the generation of initial codes, the main features of the data were identified and named systematically by exploring the latent and manifest contents. This is where line-by-line coding - reading the sentences of the transcripts, one after the other-was adopted. Another analytical procedure was 'asking questions' such as 'what is missing here?' What is happening here?; and looking for commonly used words. There was also within and between group comparison analysis of CSRCs, NCSRCs, Non-SRCs and the key informants. Bowen (2008) demonstrates that to manage data, coding can start by coding to 'broad parent codes'. This was done in the current study, enabling the breaking down of the data into manageable 'chunks'. Being that part of an extensive study is reported in this paper, efforts are made to report only part of the analytical approach relevant to the aim of the paper. However, as thematic analysis is not linear, and codes or child nodes can emerge from other 'broad parent codes' or 
'parent codes' where a different type of analytical approach was used, some analysis for other 'broad codes' are not reported.

The coding process was hybrid where the deductive approach was driven by theories in the framework of analysis. This framework of analysis was developed after an extensive literature review (Figure 1). The elements of the framework are not limited to a contextual environment comprising societal values and norms, culture, bribery and corruption, governmental and political conditions. There were, however, other codes that were data-driven hence inductive. The codes in each 'broad parent code' were arranged into a potential hierarchy, child nodes and parent codes. The reexamination of the codes was continuous. Thematic map of the visual presentation of the codes, subcategories, themes and their relationship was explored and developed; this helped sort the codes.

After the coding and collating, the themes were searched and developed by examining and arranging the codes, combining the codes to form subthemes and themes and eliminating codes. The criteria for this stage were insufficient code and data, inconsistency in data, latent and manifest meanings. The features of NVivo for Mac, text search query, Matrix coding and word tree were adopted here.

Following this, the potential themes were reviewed and refined. At level 1, the lead author read through the extracts or contents of each theme for consistency and coherency. New themes and subthemes were created, some were eliminated and some enriched. After this, the next level, 2, involved reading the entire data set against each theme for consistency and well defined to tell a compelling story. After this phase, in Phase 5, the themes were examined to ensure that they were 'fit-for-purpose'. In line with Braun and Clarke (2006), each of them captured something meaningful that will contribute to answering the research objectives. Themes that the scope and the content cannot be described in a few sentences were refined (Braun and Clarke 2006). The themes were then named, and the names of the themes sharpened, refined and not overburdened. The last phase was to write up the data and analysis to answer the research question by telling a story.

\section{RESULTS}

\section{Background information of respondents and participating organisations \\ Participating organisations}

The 13 'key informant'-Organisations with direct and indirect association and relationship with the construction industry or contractors - are not limited to two regulatory authorities (One regulator of H\&S in one state, one regulates engineering and construction practices), one higher education institution, H\&S consultancy firms and two client organisations (one public and one private). The following variables 'Constantly Self-regulating Contractors (CSRCs)', 'Non-Constantly Self-regulating Contractors (NCSRCs)' and 'Non-Self-regulating Contractors (Non-SRCs)' were used for the analysis. The CSRCs were 10 (six LCs, two MCs and one SC); the NCSRCs were 14 (one LCs, six MCs, five SCs, and two MiCs); Non-SRCs were seven (one MCs, three SCs and three MiCs). Only seven of the 10 CSRCs were part foreign and Indigenous owned and the remaining 24 were wholly indigenous owned construction companies. All the six geopolitical zones in Nigeria were represented in the sample but not in each of the three groups of analysis. The scope of operation of the 
contractors includes oil and gas, telecommunication, building, civil Engineering, renovation, and refurbishment projects. The companies operated nationwide and locally as multinationals or consultancies.

\section{Contextual effects on CSHE regulation}

Table 1 shows the summary of the contextual factors that affect the regulation of CSHE and selected resultant factors. In other words, they are indirect determinants of CSHE and the resultant factors, the direct determinants. The table shows that some of the resultant factors are repeated in more than one contextual factor because of intercontext relationships of the factors. The discourses are detailed in Figures 2 to 4 . No evidence of the contribution of the economic context was found. It was also found that not all the direct determinants of CSHE are context-based or context-resultant.

Table 1: Summary of external-context domains effects on CSHE Regulation

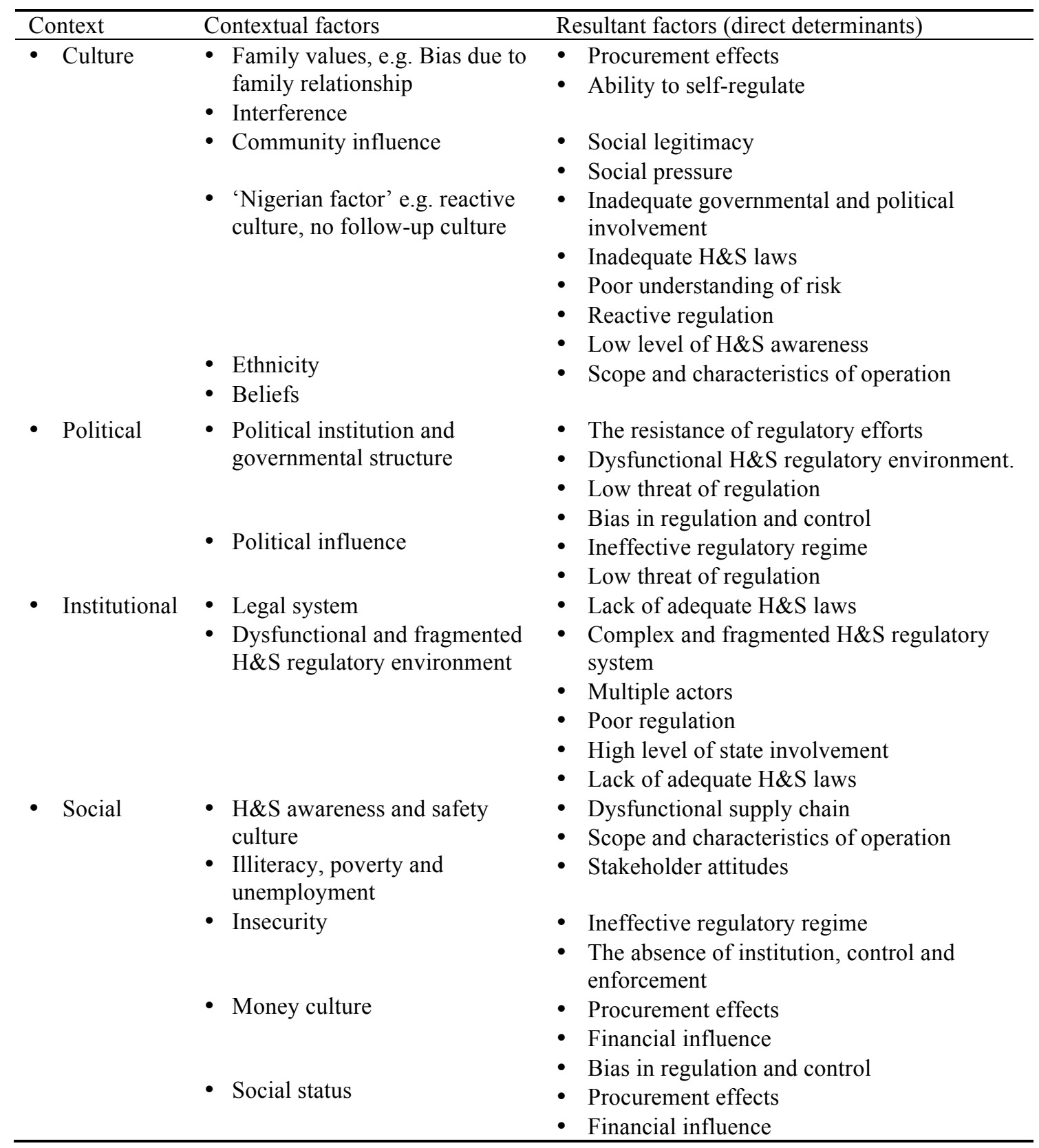




\begin{tabular}{|c|c|c|}
\hline - Religious & - Religion & $\begin{array}{l}\text { - Bias in regulation and control } \\
\text { - Procurement effect } \\
\text { - Implementation capacity } \\
\text { - Attitudes: shifting H\&S responsibility to the } \\
\text { Supernatural; } \\
\text { - H\&S is secondary. }\end{array}$ \\
\hline
\end{tabular}

\section{Cultural Context}

'Family' values: this was not found in CSRCs but in NCSRCs and Non-SRCs, characterised by family members interfering in small construction projects including $\mathrm{H} \& \mathrm{~S}$, and allocation of contracts based on family values (Figure 2). These have implications on the ability of the contractor to self-regulate. However, communities work together to ensure H\&S in communities including ensuring that H\&S considers their local context. Family in this context is beyond nuclear family; it is used as a concept here to show the close tie in the communities, see context section.

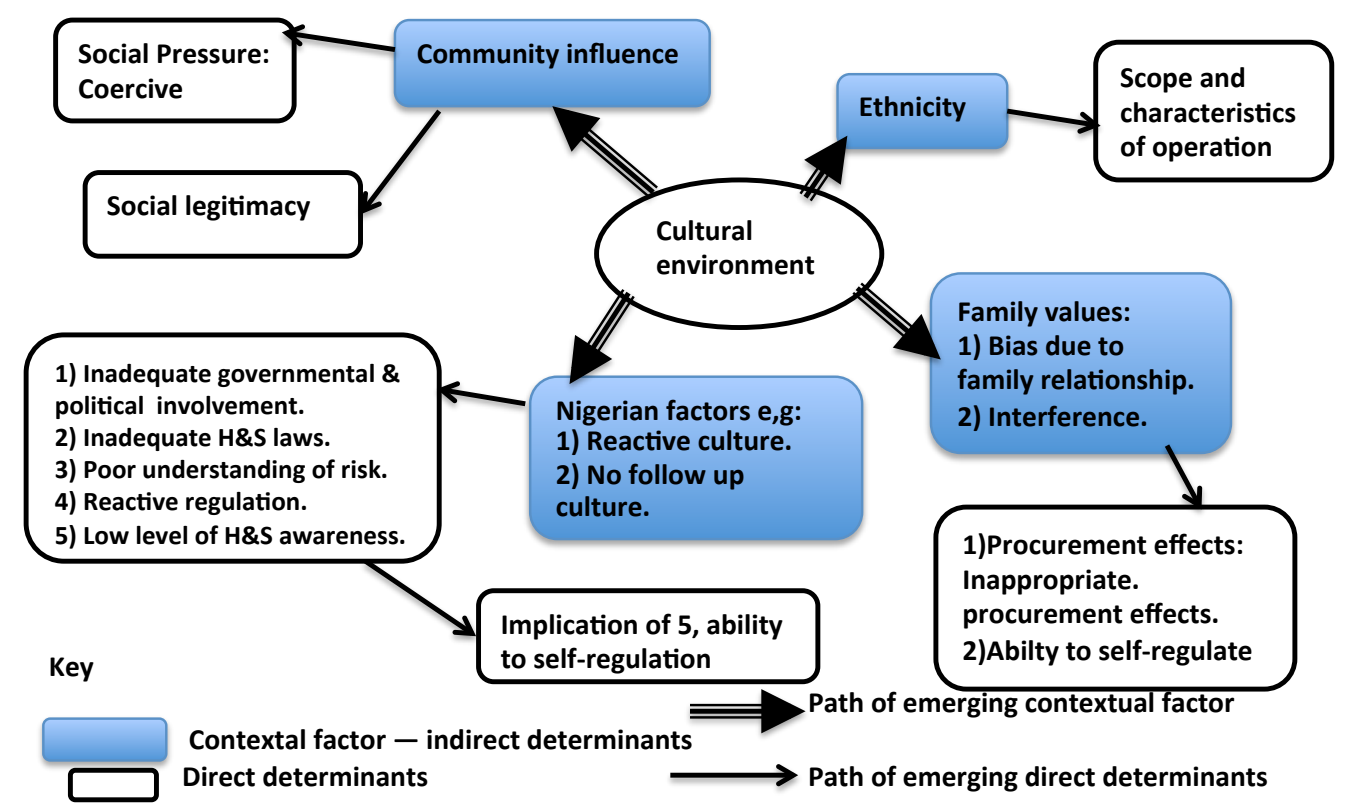

Figure 2: Summary of cultural context effects on CSHE regulation Source: Author's field work

'Nigerian factors'-issues inherent in Nigeria, turned into norms or the way of life. These were found to manifest in two ways, 'no follow-up culture' and 'reactive culture'. For 'no follow-up culture', few CSRCs and NCSRCs opine that because policies, programmes and projects are abandoned or changed as the case may be, there is a lack of continuity. Few key informants and many NCSRCs and Non-SRCs conclude that this results in inadequate H\&S laws, for instance, old laws, inadequate governmental involvement, to name but a few (Figure 2). In other words, if the reverse was the case, these implications will be less if not non-existent. Regarding the 'reactive culture' the respondents view that issues are addressed when it is late, 
measures (if applicable) are always late, after the incident; this is called 'medicine after death' in Nigeria. The respondents (NCSRCs, Non-SRCs and key informants) strongly view that efforts used in addressing H\&S issues should be proactive and not reactive. They view that this also manifests in $H \& S$ including regulatory activities.

Concerning ethnicity (Figure 2), the views of the respondents were different. While some view that this affected H\&S decisions in SMEs or projects by impacting on the level of $H \& S$, others think otherwise. There was evidence of community influence on H\&S being emphasised in the South-South of Nigeria but mainly in terms of exploiting contractors. However, there was little or no evidence of this in the South East and some Northern parts where although very minimal or negligible, the contribution of the community was positive. While Figure 2 shows pressure from the community on contractors for $\mathrm{H} \& \mathrm{~S}$, it also shows that the interests and contributions of communities in $\mathrm{H} \& \mathrm{~S}$ result in contractors self-regulating so that they become socially acceptable in society.

\section{Political Context}

The two contextual factors contribute or result in regulatory matters, on the regulator and the regulated; this includes the inter-context effects (Figure 3). The analytical groups agree to 'political influence' contributing to 'low threat of regulation' where the regulatory process and laws do not threaten the regulated because of many reasons, such as regulators unable to enforce laws on-site or companies of the people in the higher echelon of the society. This also resulted in the regulatory regime being 'ineffective and biased'. For example, one employee of a regulatory authority narrated how they were beaten and refused entry to two different sites owned by politicians on different occasions. Even when they gain access, in some sites, improvement notices they issue are ignored. Consequently, they do not bother going to such sites. This also characterises the 'insecurity' in the social environment.

'Lack of governmental and political attention' to H\&S, another contextual factor was also emphasised or suggested by the respondents, in agreement, as the core in this context (Figure 3). Many respondents suggested or noted misuse of political powers to hinder H\&S rather than drive it. One respondent typifies the thesis here:

'...the government pays no attention to H\&S except in some state such as Rivers. There is no enforcement of laws. When you go to their sites, $H \& S$ is just in paper. Did you pass the flyover at (name withheld)? What do you think about $H \& S$ there? The ironworkers are there with no harness and government officials even the governor passes there daily. Personal Protective equipment and traffic management are zero. 


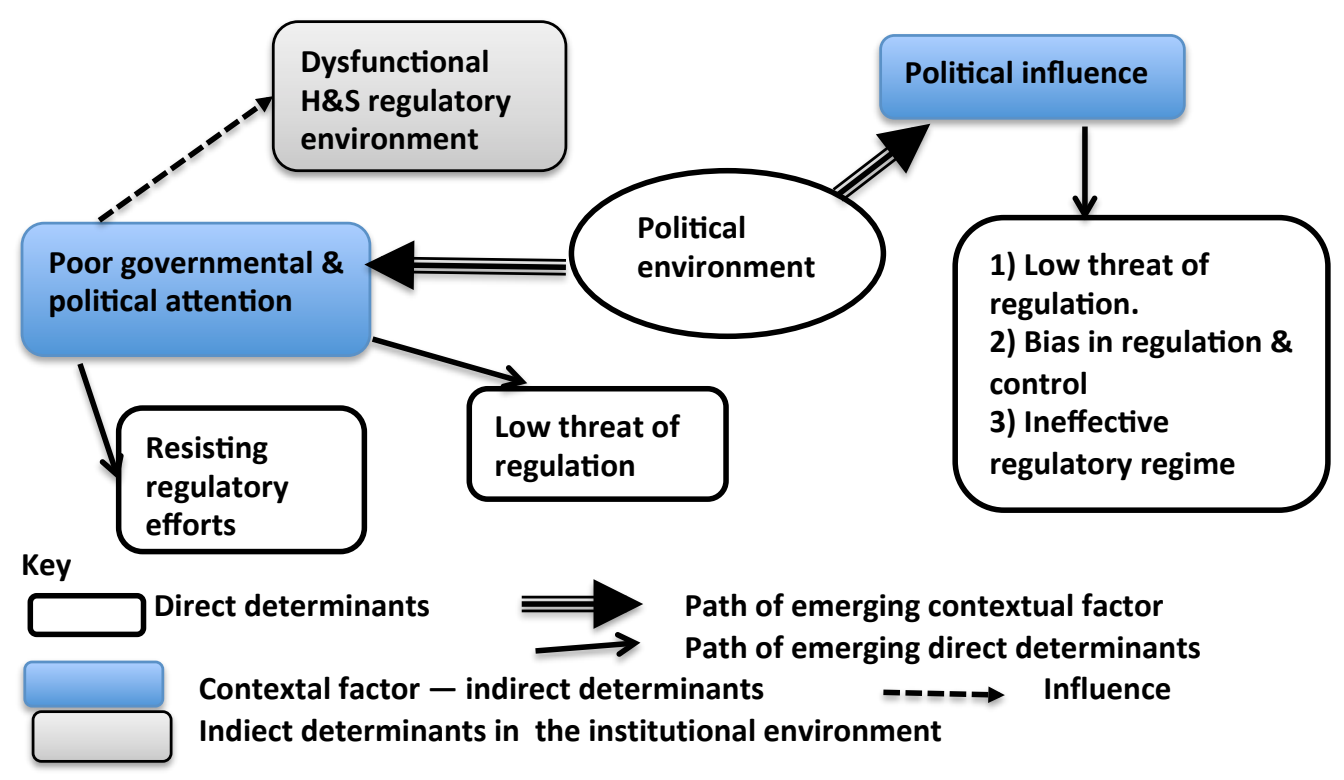

'Figure 3': Summary of political context effects on CSHE regulation Source: author's field work.

There was evidence to conclude that dysfunctional and fragmented H\&S regulatory environment is exacerbated by the lack of governmental interest in H\&S, for example, the president has not assented the H\&S bill signed since 2012, the laws are too old and not 'fit-for-purpose'; contracts are awarded with no concern for H\&S.

\section{Institutional Context}

Here, the study found that the main contextual effects come from two factors, the 'legal system' and the 'dysfunctional and fragmented H\&S regulatory environment'. Figure 4 shows that the dysfunctional and fragmented H\&S regulatory environment tends to be well experienced or emphasized in the study resulting in multiple regulatory actors such as clients, contractors, the state, and even communities; among many; high level of state involvement where some SMEs and key informants are not encouraged by the over-involvement of the state in H\&S regulation; and a heterogeneous CSHE self-regulatory regimes with different laws, interests and fragmentation. This factor contributes to the cultural context regarding the H\&S awareness and safety culture. However, the political context contributes to this factor. One respondent epitomised this thus:

When I was working for a small contractor one problem we encountered in $H \& S$ was lack of adequate enforcement.... and we do not really know what to do when we do H\&S. However, now that I work for a bigger company where $H \& S$ is taken seriously, the regulatory system is so confusing and complex. Each client or state seems to want a different thing. For example, in Lagos state, there are laws; many government authorities that tell us what to do, this is if you are even told what to do. The Lagos State Safety Commission is there; the local government is there, the Building authority is there, the environmental people are there, name them. Their expectations are sometimes contradictory. What they want is not the same as what our international clients or clients in Rivers state want. The main reason for all this is that many people tell us what to do, the standards are a lot; I would say that the problem lies in the institution. 


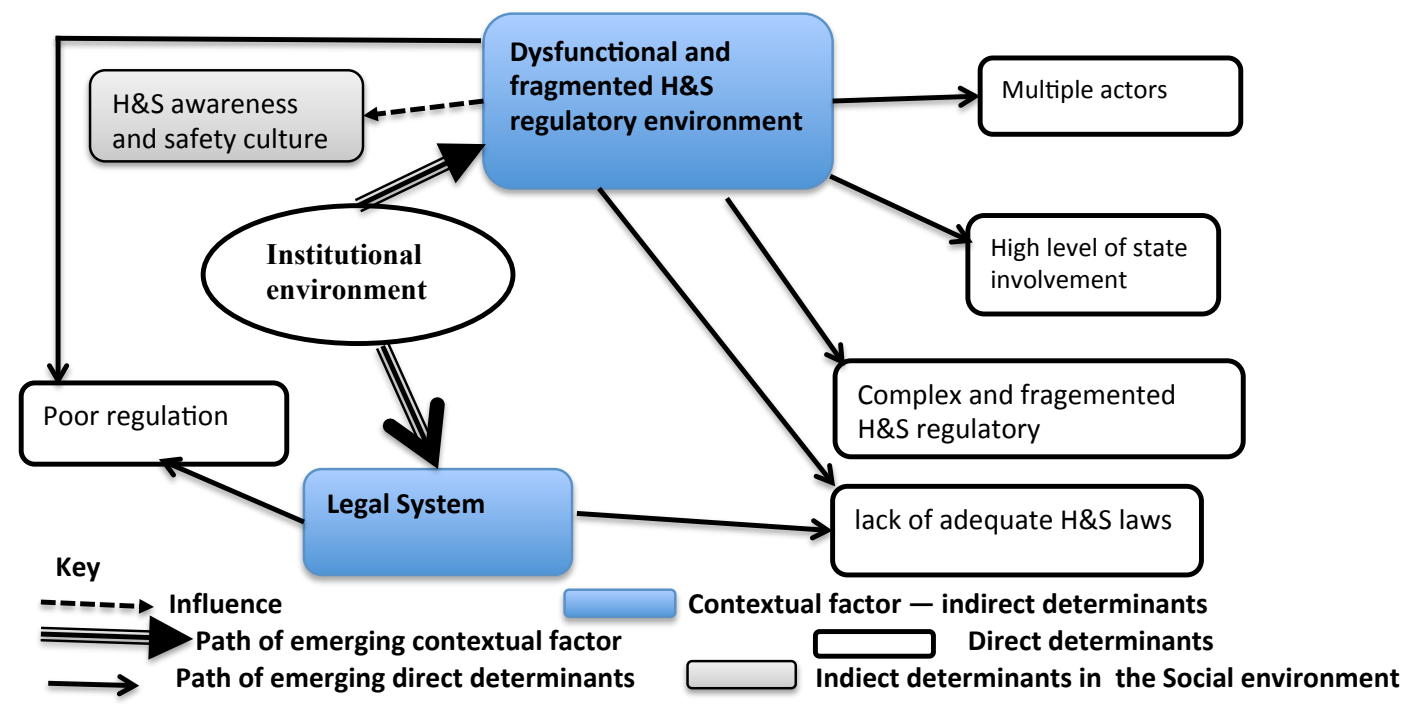

'Figure 4': Summary of institutional context effects on CSHE regulation Source: Author's fieldwork

This finding does not only show the complex nature of the regulatory system but also the risk of confusion and frustration and operational constraints. The evidence in the study shows that 'poor regulation of H\&S' and 'lack of adequate H\&S laws' are the two main factors in the Institutional Environment context. The lack of adequate judicial system does not only result in lengthy court cases but encourages noncompliance and lack of enforcement. From Figure 4, it can be argued that the complexity of CSHE regulation tends to be rooted here in that the resultant factors (Direct determinants) are fundamental for improving H\&S.

\section{Social and religious contexts}

Insecurity: while this has been noted in political context, it also manifested here, but different in that the communities use means such as kidnapping, abuse of contractors to ensure that the demands (including those under the guise of $\mathrm{H} \& \mathrm{~S}$ ) are met. 


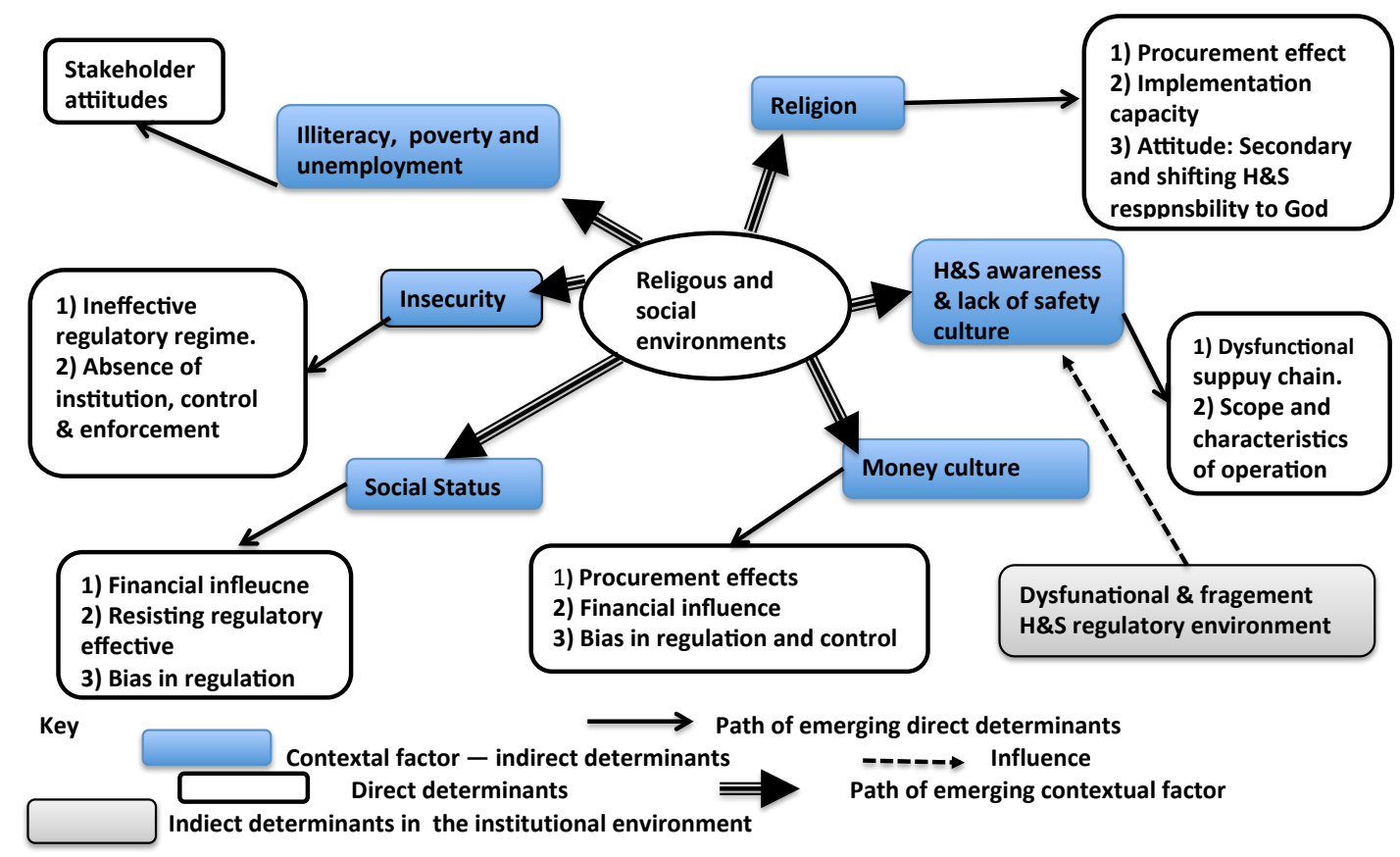

Figure 5: Summary of social and religious context effects on CSHE regulation Source: authors' fieldwork

Illiteracy, poverty and unemployment: this manifested in various ways (see Figure 5). The construction industry is characterised by high level of illiteracy, both from the client and, contractors and workers and poverty, according to few CSRCs, NCSRCs and key informants. This results in the lack of knowledge on the part of clients and workers where the clients do not engage in $\mathrm{H} \& \mathrm{~S}$ because of this and workers do not know their rights. Some organisation and even the government then make a conscious decision to disregard the unsafe working conditions and the safety of the public.

H\&S awareness and safety culture: there was evidence to conclude that this is emphasised in the industry. The four groups analysed highlighted the implication of low level of H\&S awareness, but the groups experience this differently. People with a poor understanding of H\&S mainly exploit the CSRCs hence do not support H\&S, but the NCSRCs struggled to convince clients with a poor understanding of H\&S to support it. The Non-SRCs are also faced with this, but would instead get the contracts than pushing the client for H\&S. It can be inferred that the supply chain does not function, as it should.

Money culture: There is evidence to conclude the general belief in the industry of money being at the 'heart' of every activity; it is viewed as the solution to all things. This then makes a recipe or platform for corruption experienced in procurement and regulatory practices.

Social status: the position of people in the society affect regulatory activities in that the state regulator of $H \& S$ is biased in favour of contractors in the high echelon of the society or their connections. A respondent states as follows:

Without putting a stop to 'man know man', the problems in Nigeria will still be there Once you are 'connected', you are above the law. Nobody can touch you. One top government official that has a project close to my house is building 
without approval, but nobody will touch him. If they are so strict, they will give him 'executive approval' if they pay the right fees. However, the 'common man' will have to pay through his nose and go through a very stressful approval process.

Conversely, there were indications of people in the high echelon of the society supporting and even driving H\&S through various ways. Secondly, there was evidence that that ability of contractors or clients to affect or resist regulatory activities due to their political or social status inform their decision to self-regulate.

Religion: there was no consensus on this influencing H\&S, as few CSRCs, NonSRCs, NCSRCs and key informants agree to this, but few NCSRCs and key informants view otherwise. This manifests in ways such as determining the attitudes and behaviours of workers. Few Non-SRCs and NCSRCs claimed to have experienced where particular religious groups are preferred over others hence awarded contracts. Few respondents claim to have experienced where lower standards or even complete overlook of poor H\&S practices during enforcement and monitoring of H\&S internal standards and policies are in favour of people of the same religion. There is evidence of shifting the locus of control to the supernatural-transferring $\mathrm{H} \& \mathrm{~S}$ to the supernatural.

\section{DISCUSSIONS}

The context-informed, careful and purposeful selection of respondents and organisations enabled the depiction of the full breadth of the population for qualitative research (Adami 2005) meeting what Patton (1990) calls information-rich cases. Hence, the respondents provided great information that is fully context-based. Some of the CSRCs are indigenous contractors, but have a few things in common, for example, the scope of operation including the type of location and nature of client and operations.

The findings of the study offer valuable insight into the eternal-context domains of Nigeria and connections with the regulation of CSHE including the external-context determinants of CSHE regulation. One of the key factors is the 'dysfunctional and fragmented H\&S regulatory environment' due to poor governmental and political attention to H\&S. Drawing on Nuwayhid (2004) and the introductory section, this is consistent with extant literature in developing and emerging countries in that the barrier to occupational health lacks governmental interest, adequate data, and poor enforcement of laws. According to the findings of Kheni (2008), the government even fails to show good examples in their project. 'The worse abuse of health, safety and welfare can be found on construction sites of government-funded projects which can be traced to lack of effective implementation of policies relating to occupational health and safety' (Kheni 2008: 200).

Most developing countries lack the political mechanism that enables putting information and policies into action (Nuwayhid 2004). This is consistent with the findings of the current study where 'Nigerian factors' 'no follow-up culture' and 'reactive culture' are reported as culture context factors for CSHE regulation (Figure 2 ). Further, the strong cultural values of developing countries where cultural values such as family values positively affect $\mathrm{H} \& \mathrm{~S}$ practices are reported in Ghana (Kheni 2008) and Nigeria (Umeokafor 2018); the points in the 'Context' section explain this. 
In the current study, its effect is also reported in terms of procurement and interference in small construction projects. While domestic clients or small project clients are not accountable to anyone on whom to award contracts or the criteria for this, contracts awarded based on family values are likely to hinder competition and $\mathrm{H} \& \mathrm{~S}$. This is because the H\&S competency and experience of the contractor (who is awarded the contracts based on family values) may not be assessed or poorly assessed. These have implications on the ability of the contractor to self-regulate. However, the positive implications of community involvement in H\&S are encouraging.

Nigeria, being a religious nation, the findings in this regard is expected and has been reported in studies such as Umeokafor (2015) in Nigeria and by Kheni (2008) in Ghana. The impact or effect of this on the risk perception of the respondents shows the importance of considering not only culture in risk perception but also religiousness. Religion is core in developing countries and cannot be treated in isolation when addressing issues in developing and emerging economies.

In the opinions of the respondents, while the communities are involved in $\mathrm{H} \& \mathrm{~S}$, the geographic difference in the attitudes of the communities is consistent with the literature. Umeokafor (2018b) found that geographic locations are likely to determine community intervention in $\mathrm{H} \& \mathrm{~S}$ which impact on the attitude toward $\mathrm{H} \& \mathrm{~S}$, its performance and activities. The views of the respondents on communities exploiting contractors are consistent with Umeokafor (2018b). However, Umeokafor (2018a) reports on other positive roles and implications of a community intervention in $\mathrm{H} \& \mathrm{~S}$ - defining H\&S standards and requirements and contextualising H\&S.

Literature discussion shows that enforcement officers are compromised through bribery and that there is discrimination in the enforcement of H\&S laws. These are consistent with the findings of the current study. This shows the need for measures for transparent regulation and other activities in construction. Corrupt activities are reported in the construction industry in general, specifically, in Ghana (Kheni 2008), Nigeria (Umeokafor 2018), in the UK (Teodorescu, 2016). The money culture may explain this level of corruption in the country, or as Odole (2018) puts it, 'a culture of bribery'.

The introductory section and a review of ILO (2017) show that the Nigerian construction industry deals with many regulatory actors and many laws, suggesting the distributed nature of the regulatory environment that Abubakar (2016) discusses. This is consistent with the findings of the current study and one of the key determinants of the complex CSHE regulatory environment.

\section{IMPLICATIONS FOR RESEARCH AND PRACTICE}

Overall, the study provides insight into how complex CSHE regulatory regimes can be affected by the external-context domains of the countries which regulatory authorities, academics, socio-legal scholars/practitioners and the construction industry may find helpful. The implications of the study for practice and research include:

While the data is from Nigeria, other developing and emerging economies may benefit from the findings in that they can serve as a framework for more country-specific studies. For developed countries, the study can be a contrasting standard for them. 
The external-context domain of countries is inextricably linked to the complex regulatory environment in terms of CSHE. In developing a robust and pragmatic $\mathrm{H} \& \mathrm{~S}$ regulatory environment, the effects of the social, political, institutional, cultural and religious context need to be considered and factored in any strategies. Indeed, the attention of the government and politicians seems to be the pivot for the dysfunctional and fragmented CSHE regulatory environment. The continuity in policies and strategies is needed. In the absence of this, strategies that will get the government involved in $\mathrm{H} \& \mathrm{~S}$ with the political will to sustain H\&S development is needed.

The need for a consolidated regulatory framework for H\&S with fewer regulatory actors and adequate contextualised CSHE legislation is needed. The need for an unbiased regulatory system that would establish and sustain a high level of regulatory threat is also evident.

There is the need for a regulatory framework that is resilient to political effects and social-status effects. However, the potential contributions of social parties or authorities to H\&S and their connection or ties and effects on the society need to be harnessed to the advantage of H\&S.

\section{CONCLUSIONS}

The study explored and explained the contextual effects on CSHE regulation, from the Nigerian perspective. The study established that external-context domain of countries is inextricably linked to the complex CSHE regulatory regime of EDEs. There is evidence that the external-context domain factors are secondary factors of CSHE regulation of which some main ones include the dysfunctional and fragmented $\mathrm{H} \& \mathrm{~S}$ regulatory environments, which is exacerbated by the poor governmental and political attention on H\&S. 'Nigeria factors' such as the 'reactive culture' and 'no follow-up culture' are among the indirect factors that result in inadequate governmental and political involvement, among many, poor regulation and inadequate H\&S laws. There is a low threat of regulation, a direct factor, of which one of the political environment factors includes the political influence and the 'legal system' that underperforms. Based on the findings of the study, a contextualised and consolidated H\&S regulatory framework that is resilient to social and political pressure that will provide unbiased regulation are recommended. The ties of the social and cultural authorities with the society and the effects on them should be exploited to the advantage of $H \& S$, for example by involving contractors in $H \& S$ policymaking.

\section{REFERENCES}

Abubakar, .U. (2016) Structural and implementation issues around the new Nigerian Labour, Safety, Health And Welfare Bill (2012): Lessons From UK, USA, Australia And China. Transactions of the VŠB - Technical University of Ostrava, Safety Engineering Series, Vol. 11 (1), pp. 61-71.

Adami M.F. (2005) The use of triangulation for completeness purposes. Nurse Researcher 12(4), 19-29.

Alggaia and Millington (2008) Male Child Sexual Abuse: A phenomenology of betrayal. Clinical Social Work 36, 265-275 
Alkilani, S. Z., Jupp, J. and Sawhney, A. (2013) 'Issues of construction health and safety in developing countries: a case of Jordan', Australasian Journal of Construction Economics and Building, 13 (3) 141-156

AlSehaimi, A., Koskela, L., and Tzortzopolulos, P. (2013). "Need for alternative research approaches in construction management: case of delay studies". Journal of Management in Engineering, 29 (4), 407-413.

Bowen, G.A. (2008), "Naturalistic inquiry and the saturation concept: a research note", Qualitative Research, Vol. 8 No. 1, pp. 137 -152.

Braun, V. and Clarke, V. (2006) Using thematic analysis in psychology, Qualitative Research in Psychology, 3:2, 77-101.

Brod, M., Tesler, L.E. and Christensen, T.L. (2009) Qualitative research and content validity: Developing best practices based on science and experience. Quality of Life Research, 18, 1263-1278.

Castillo-Montoya, M. (2016) Preparing for Interview Research: The Interview Protocol Refinement Framework. The Qualitative Report, 21 (5) 811-831.

Creswell, J. W. (2009), Research Design: Qualitative, Quantitative and Mixed Method Approaches, 3rd ed, Sage, California.

Creswell, J. W. and Miller, D. L. (2000) Determining validity in qualitative inquiry, Theory into Practice, 39(3), 124-130.

Darwish, A. E., and Huber, G. L. (2003). "Individualism vs Collectivism in Different Cultures: A cross-cultural study", Intercultural Education, 14(1), 47-56.

Diugwu I.A., Baba D. L., and Egila A. E. (2012) Effective regulation and level of awareness: An expose of Nigeria's construction industry. Open Journal of Safety Science and Technology, 2012, (2), pp 140-146

Erikkson, P. and Kovalainen, A. (2008) Qualitative research in Business research. Thousand Oak. CA. Sage publications.

Fereday, J. and Muir-Cochrane, E. (2006) Demonstrating rigor using thematic analysis: A hybrid approach of inductive-deductive coding and theme development. International Journal of Qualitative Methods 5(1) 1-11.

Finneran, A and Gibb, A. (2013) W099: Safety and Health in construction: Research Roadmap report for consultation. Retrieved on 05-08-2018 from https://dspace.lboro.ac.uk/dspace-jspui/bitstream/2134/12523/3/pub\%20376.pdf

Gunningham, N. (2011) Investigation of industry self-regulation in workplace health and safety in New Zealand. Gunningham \& Associates Pty Ltd. Retrieved on 16-07-18

from http://regnet.anu.edu.au/sites/default/files/publications/attachments/201504/NG_investigation-industry-self-regulation-whss-nz_0.pdf

Gunningham, N. and Rees, J. (1997) Industry Self-Regulation: An Institutional Perspective, Law \& Policy, Vol. 19, (4),

Gutiérrez, L., GlenMaye, L., and DeLois, K. (1995) The organizational context of empowerment practice: Implications for social work administration. Social Work,40, (2), 249-258.

Health and safety executive (HSE) (2015), Managing health and safety in construction: Construction Design and Management, Retrieved on 06-07-18 from http://www.hse.gov.uk/pubns/priced/1153.pdf

Health and Safety Executive (HSE) (2018) Workplace fatal injuries in Great Britain 2018. Retrieved on 10 July from http://www.hse.gov.uk/statistics/pdf/fatalinjuries.pdf

Hofstede, G. (2001). Culture's consequences; Comparing values, behaviors, institutions, and organizations across nations, Sage Publications, London. 
Humble, A. M. (2009) Technique triangulation for validation in directed content analysis. International Journal of Qualitative Methods, 8(3), 34-51.

Idoro, G.I. (2011) Comparing occupational health and safety (OHS) management efforts and performance of Nigerian construction contractors. Journal of Construction in Developing Countries, 16(2), 151-173.

International Labour Organisation (ILO) (2017) Nigeria Country Profile on Occupational Safety and Health 2016. Retrieved on 27 August 2018 from https://www.ilo.org/wcmsp5/groups/public/---africa/---ro-addis_ababa/---iloabuja/documents/publication/wcms_552748.pdf

Isaacs, A. N. (2014) An overview of qualitative research methodology for public health researchers. International Journal of Medicine and Public Health, 4(4), 318- 323.

Kheni, N A, Gibb, A G F and Dainty, A R J (2010) The health and safety management within small and medium-sized enterprise (SMEs) in developing countries: Study of contextual influence.'Journal of Construction Engineering and Management',136(10), $1104-1115$

Kheni, N.A. (2008), "Impact of health and safety management on safety performance of small and medium-sized construction business in Ghana", Doctoral thesis, Loughborough University, available at: https://dspace.lboro.ac.uk

Kakkuri-Knuuttila, M.-L., Lukka, K., \& Kuorikoski, J. (2008). Straddling between paradigms: A naturalistic philosophical case study on interpretive research in management accounting. Accounting, Organizations and Society, 33(2\&3), 267-291.

Lincoln, Y. S. and Guba, E. G. (1985). Naturalistic Inquiry. Newbury Park, CA: Sage Publications.

Lukka, K. (2014) Exploring the possibilities for causal explanation in interpretive research. Accounting, Organisations and Society, 39, 559-566.

Manu, P., Mahamadu, A., Phung, V. M, Nguyen, T. T, Chandavid Ath, Heng, A. Y. T., and Kit, S. C (2018) Health and safety management practices of contractors in South East Asia: A multi country study of Cambodia, Vietnam, and Malaysia. Vol.107, 188-201, https://doi.org/10.1016/j.ssci.2017.07.007

Maxwell, J. A. (2004) Using qualitative methods for causal explanation. Field Methods, 16 (3) 243-264

McCracken, G. (1988) The long interview. Newbury Park, California: SAGE Publications.

MOLISA (2012) National programme on occupational safety and occupational health in period of 2011-2015. MOLISA. Available https://www.ilo.org/wcmsp5/groups/public/---asia/---ro-bangkok/---srobangkok/documents/policy/wcms_186680.pdf (Accessed 21/08/2018).

Muiruri, G. and Mulinge, C. (2014) Health and Safety Management on Construction Projects Sites in Kenya: A Case Study of Construction Projects in Nairobi County. FIG Congress 2014, Engaging the Challenges - Enhancing the Relevance, Kuala Lumpur, Malaysia 16-21 June.

Nuwayhid, I. M. (2004) Occupational Health Research in Developing Countries: A Partner for Social Justice. American Journal of Public Health. 2004 November; 94(11): 1916-1921.

Odole, E., B. (2018) Factors that Encourage Unethical Practices by Organizational Leaders in Nigeria. Doctoral Thesis, Retrieved on 01 Sept 2018 from https://scholarworks. waldenu.edu/cgi/viewcontent.cgi? article $=6391 \&$ context $=\mathrm{di}$ ssertations 
Okojie, O. (2010). System for reporting occupational diseases in Nigeria. African Newsletter on Occupational Health and Safety, 20 (3), 51-53.

Okoye, P. U. (2018) Occupational Health and Safety Risk Levels of Building Construction Trades in Nigeria. Construction Economics and Building, 18:2, pp. 92-109

Omeife, C. A. and Windapo, O. A. (2013) The impact of national building code on professionalism. Proc.: 43rd Builders Conference/AGM, Abuja, Nigeria, 1-12.

Palys, T. (2008) Purposeful sampling. In L. M. Given (Ed.) The Sage Encyclopedia of Qualitative Research Methods. (Vol. 2). Sage: Los Angeles, 697-8.

Patton, M. (1990) Qualitative evaluation and research methods (169-186). Beverly Hills, CA: Sage.

Pew Forum (2010) Appendix B: Religious demography of Sub-Saharan Africa. http://www.pewforum.org/files/2010/04/sub-saharan-africa-appendix-b.pdf [23 January 2018].

Plumper, T., Troeger, V. E., and Neumayer, E. (2010) Case Selection and Causal Inference in Qualitative Research. Available at SSRN: https://ssrn.com/abstract=1439868 or http://dx.doi.org/10.2139/ssrn.1439868

Pybus, R (1996) Safety Management: Strategy and Practice. Oxford: ButterworthHeinemann.

Sands, R. G. and Roer-Strier, D. (2006) Using data triangulation of mother and daughter interviews to enhance research about families. Qualitative Social Work 5(2), 237-260.

Saunders, M., Lewis, P. and Thornhill, A. (2009) Research methods for business students (5th edition), Prentice-Hall, London UK

Teodorescu, L. (2016), "Five years on: what impact has the bribery act had on construction?", Construction Manager, pp. 38-39.

Umeokafor, N. and Windapo, A. (2018) Challenges to and opportunities for establishing a qualitative approach to Built Environment research in higher education institutions, Journal of Engineering, Design and Technology, https://doi.org/10.1108/JEDT-06-2017-0057

Umeokafor, N. I. (2018a) Construction health and safety research in Nigeria: towards a sustainable future. In Saurin, T. A., Costa, D. B., Behm. M., and Emuze, F. (Eds.). Proc. Joint CIBW99 and TG59 Conference, Salvador, 213-221

Umeokafor, N. I. (2018b) Community interventions in construction health and safety and its implications: Evidence from Nigeria. Journal of Financial Management of Property and Construction, 23 (3), DOI: 10.1108/JFMPC-10-2017-0041.

Umeokafor, N. I., and Isaac, D. (2015). A framework for analysing the determinants of health and safety self-regulation in the construction industry. In M. Behm \& McAleenan (Ed.), Proc.: CIB W099 International Health and Safety Conference: Benefitting Workers and Society Through Inherently Safe Construction, 10-11 September, Northern Ireland, 478-487.

Umeokafor, N. I., Windapo, A. O., and Manu, P. A. (2018) Country context-based opportunities for improving health and safety. In Saurin, T. A., Costa, D. B., Behm. M., and Emuze, F. (Eds.). Proc. Joint CIBW99 and TG59 Conference, Salvador, 177-186

Umeokafor, N.I. (2015) An assessment of the influence of contextual environment on health and safety practices in the Nigerian construction industry. In Behm, M. and McAleenan, C (Ed.), Proc.: CIB W099 International Health and Safety Conference, Northern Ireland, 397-406, 
Windapo, A. (2013) Relationship between degree of risk, cost and level of compliance to occupational health and safety regulations in construction. Australasian Journal of Construction Economics and Building, 13 (2) 67-82

Worldatlas (2018). Largest Ethnic Groups In Nigeria, https://www.worldatlas.com/articles/largest-ethnic-groups-in-nigeria.html $\quad[13$ August 2018]. 